Huerta, A., Vidal, Y. and Bonet, J., Updated Lagrangian formulation for corrected Smooth Particle Hydrodynamics, International Journal of Computational Methods, Vol. 3, Issue 4, pp. 383-399, 2006

\title{
UPDATED LAGRANGIAN FORMULATION FOR CORRECTED SMOOTH PARTICLE HYDRODYNAMICS
}

\author{
ANTONIO HUERTA, YOLANDA VIDAL \\ Departament de Matemàtica Aplicada III, Laboratori de Càlcul Numèric (LaCàN), \\ Universitat Politècnica de Catalunya, \\ Jordi Girona 1, Barcelona, E-08034,Spain \\ \{antonio.huerta,yolanda.vidal\}@upc.es \\ http://<www-lacan.upc.es $>$ \\ JAVIER BONET \\ Civil and Computational Engineering Center $\left(C^{2} E C\right)$, \\ University of Wales Swansea \\ Singleton Park, Swansea, SA2 8PP, United Kingdom \\ j.bonet@swansea.ac.uk
}

\begin{abstract}
Smooth Particle Hydrodynamics (SPH) are,in general, more robust than finite elements for large distortion problems. Nevertheless, updating the reference configuration may be necessary in some problems involving extremely large distortions. If a standard updated formulation is implemented in SPH zero energy modes are activated and spoil the solution. It is important to note that the updated Lagrangian does not present tension instability but only zero energy modes. Here an stabilization technique is incorporated to the updated formulation to obtain an improved method without mechanisms.
\end{abstract}

Keywords: Corrected SPH; total Lagrangian formulation; large deformations.

\section{INTRODUCTION}

In its original form Smooth Particle Hydrodynamics (SPH) had several weak points, described in detail by Swegle et al. [1995] and Belytschko et al. [2000]. These problems consisted, among others, on lack of consistency, tension instability and the presence of zero energy modes in the numeric solution.

The correction of SPH in order to reproduce polynomials in finite domains as well as passing the patch test has been an area of intensive work. Some of these contributions, without being exhaustive, are discussed in this paper. See [Liu et al. (2003)] for a detailed discussion on reproducibility of SPH methods or [Huerta et al. (2004)] for a general review of meshfree methods. Johnson and Beissel developed the normalized smoothing method [Johnson and Beissel (1996); Johnson et al. (1996)] obtaining linear consistency. Chen et al. [1999] introduce a corrected kernel by 
invoking a Taylor series expansion. Bonet and Kulasegaram [2000] developed the Corrected Smooth Particle Hydrodynamics (CSPH) method that allows to obtain linear consistency in the interpolation of the function and in the interpolation of the gradient. Consistency is achieved introducing corrections in the kernel functions and in their derivatives.

The classical SPH formulation defining a fixed support in the laboratory for each particle and thus recomputing neighbors at each time-step (i.e. updated neighbor search) that will be called here Eulerian formulation presents tension instability, see for instance [Monaghan (1982)]. Nevertheless, Bonet and Kulasegaram [2001] show that a (total) Lagrangian formulation removes this instability. It is important to note however that zero energy modes still remain in the Lagrangian formulation. Without tension instability, a SPH Lagrangian formulation presents serious advantages compared to finite elements. For instance, Libersky et al. [1993] applied successfully the Lagrangian SPH code to high strain problems, Johnson et al. [1993] incorporated the SPH algorithm into a standard Lagrangian code such as EPIC and Stellingwerf and Wingate [1993] as well as Johnson et al. [1996] used SPH for impact

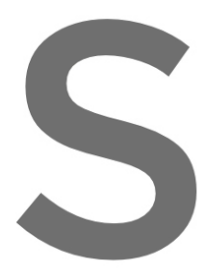
problems. Nevertheless
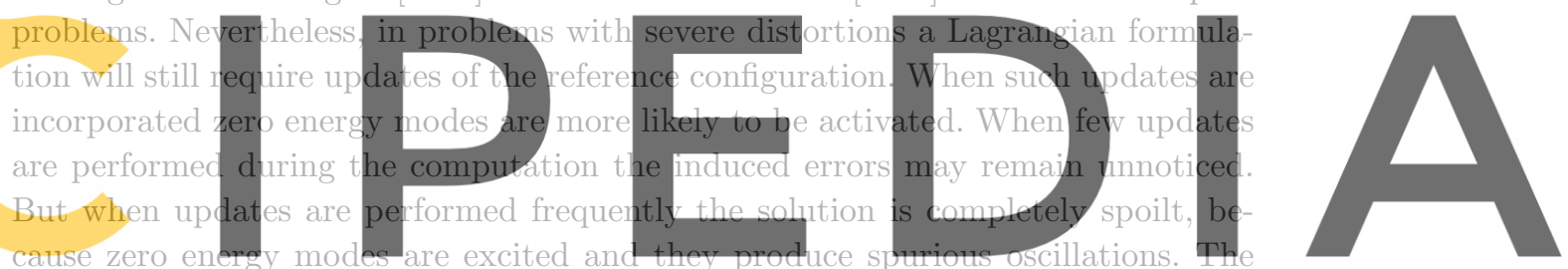

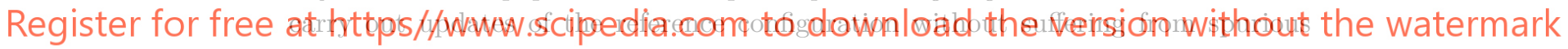
modes.

The problem of zero energy modes is still open. In the literature two types of solutions are used: dissipate spurious modes (conceptually similar to the techniques used in finite elements for hourglass control [Flanagan and Belytschko (1981)]) or an alternative discretization that does not evaluate the variables and their derivatives at the same points. For example, Gray et al. [2001] precluded the instability introducing an artificial stress (but that introduces also small errors in the solution) and Randles and Libersky [2000] used different sets of particles to interpolate different fields generating the denominated stress points. Here, for computational efficiency, particles are the only information carrying points.

First, the total Lagrangian CSPH formulation is revised for large strains dynamic problems and a numerical example is performed. Then, in section 3 the standard updated Lagrangian formulation is recalled and analyzed to show its drawbacks in SPH. Finally in section 4 a stabilized updated Lagrangian formulation is proposed and its performance is assessed both in a simple one-dimensional synthetic example and in a numerical benchmark test extremely sensible to zero energy modes. 


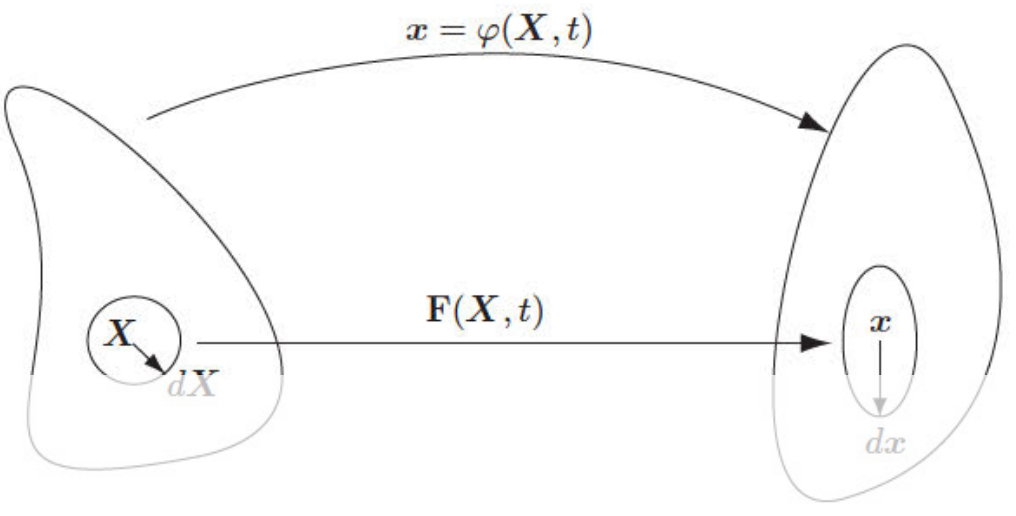

Fig. 1. Movement of a deformable body.

\section{TOTALLY LAGRANGIAN CSPH}
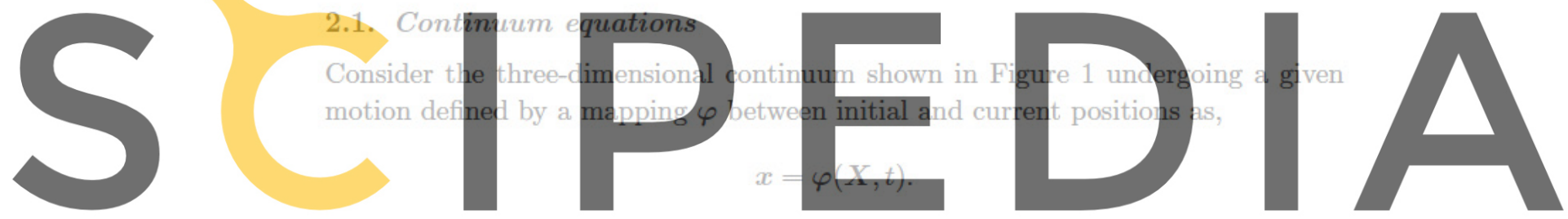

The deformation gradient $\mathrm{F}$, defined as

Register for free at https//www.scipedia.com to download the version without the watermark

is a quantity of interest in the study of large deformations because it is present in all those equations that relate magnitudes in the initial configuration with their corresponding ones in the final configuration. The volume change of the continuum can be obtained in terms of the Jacobian

$$
J=\operatorname{det} \mathbf{F}=\frac{d V}{d V^{0}}
$$

where $d V^{0}$ and $d V$ represent the initial and current element volumes. The Cauchy equation (balance of momentum equation) for the deformable body reads

$$
\nabla \cdot \sigma+\zeta f=\zeta a
$$

where $\zeta$ is the material density, $\boldsymbol{a}$ is the acceleration, $\boldsymbol{f}$ is external force per unit of volume and $\sigma$ is the Cauchy stress tensor. Recall that,

$$
\mathbf{P}=J \sigma \mathbf{F}^{-T},
$$

where $\mathbf{P}$ is the first Piola-Kirchhoff tensor. 


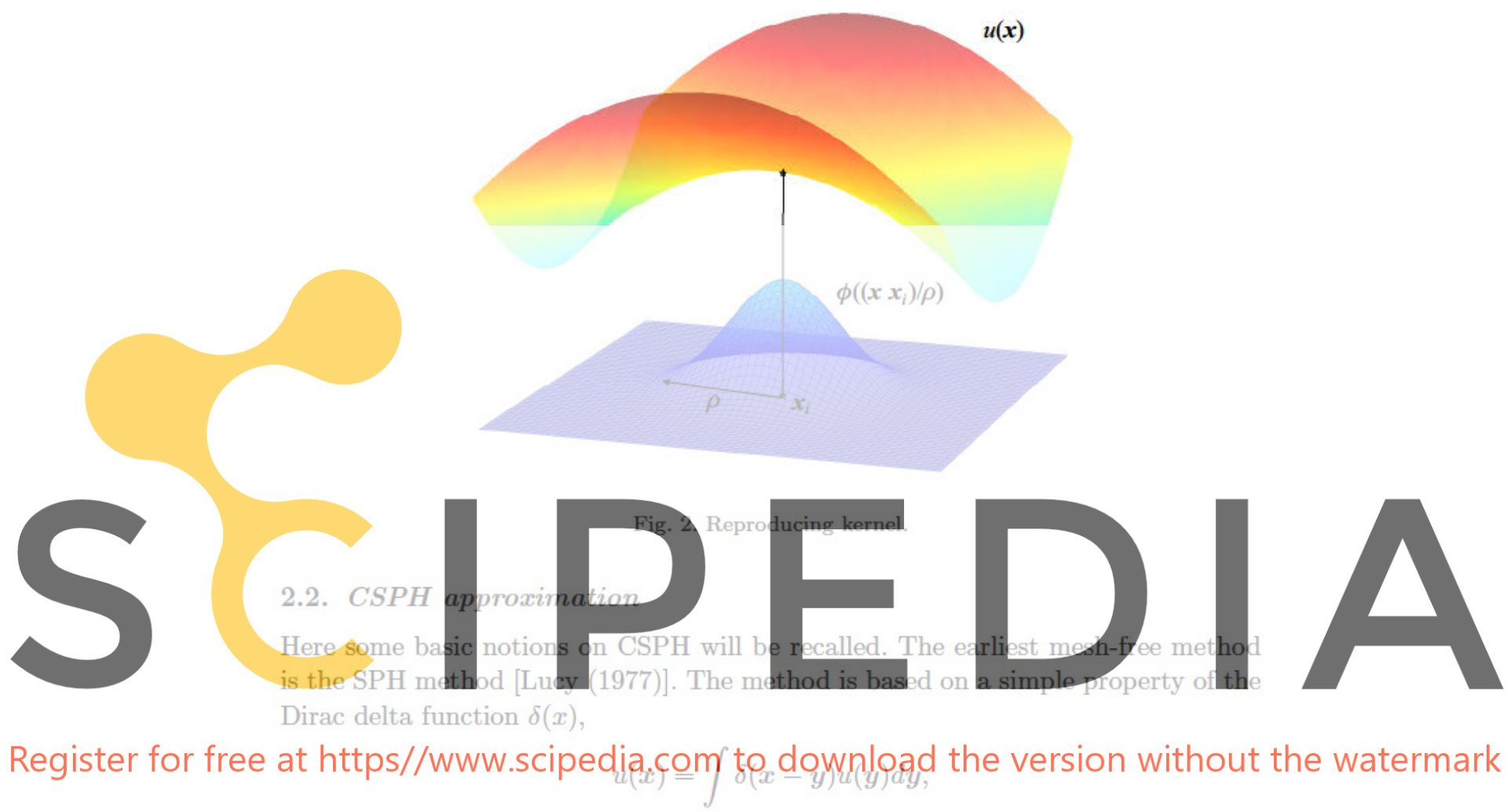

where $u(x)$ is the function to be approximated. The key idea is to replace the Dirac delta function by a kernel or weight function $C_{\rho} \phi((x-y) / \rho)$ positive, even and compact supported, see Figure 2,

$$
u(\boldsymbol{x}) \simeq \widetilde{u}^{\rho}(\boldsymbol{x}):=\int C_{\rho} \phi\left(\frac{\boldsymbol{x}-\boldsymbol{y}}{\rho}\right) u(\boldsymbol{y}) d \boldsymbol{y},
$$

where $\rho$ is called the dilation parameter, which is usually the support radius of the kernel function, and $C_{\rho}$ is a normalization constant such that

$$
\int C_{\rho} \phi\left(\frac{x-y}{\rho}\right) d y=1
$$

i.e. constant functions are exactly interpolated. Therefore, as $\rho$ tends to zero the kernel function approaches the Dirac delta function, and consequently,

$$
\lim _{\rho \rightarrow 0} \widetilde{u}^{\rho}(x)=u(x) .
$$

In order to develop a computational technique, it is necessary to evaluate the integration in equation (3) in a discrete manner to give

$$
u(x) \simeq \widetilde{u}^{\rho}(x) \simeq u^{\rho}(x):=\sum_{i} V_{i} C_{\rho} \phi\left(\frac{x-x_{i}}{\rho}\right) u\left(x_{i}\right),
$$


where $\boldsymbol{x}_{i}$ and $V_{i}$ are the points and weights of the numerical quadrature. Usually the quadrature points are called particles and the weights are called volumes.

It is possible to re-write equation (4) in terms of standard shape function as

$$
u(\boldsymbol{x}) \simeq u^{\rho}(\boldsymbol{x}):=\sum_{i} N_{i}(\boldsymbol{x}) u\left(\boldsymbol{x}_{i}\right), \quad N_{i}(\boldsymbol{x})=V_{i} C_{\rho} \phi\left(\frac{\boldsymbol{x}-\boldsymbol{x}_{i}}{\rho}\right) .
$$

As a result of point integration in equation (4), the consistency conditions are no longer satisfied exactly. Bonet and Kulasegaram [2000] present a corrected SPH approximation (CSPH) to preclude these difficulties. The foregoing is a brief review of the three main corrections introduced by Bonet and coworkers.

First, the discrepancy in the numerically integrated consistency conditions is eliminated by a kernel correction. As proposed by Liu et al. [1995], $C_{\rho}$ is selected by enforcing linear consistency conditions now given by a point-wise integration as,

$$
\sum_{i} V_{i} C_{\rho} \phi\left(\frac{x-x_{i}}{\rho}\right)=1, \quad \sum_{i} V_{i}\left(x-x_{i}\right) C_{\rho} \phi\left(\frac{x-x_{i}}{\rho}\right)=0 .
$$
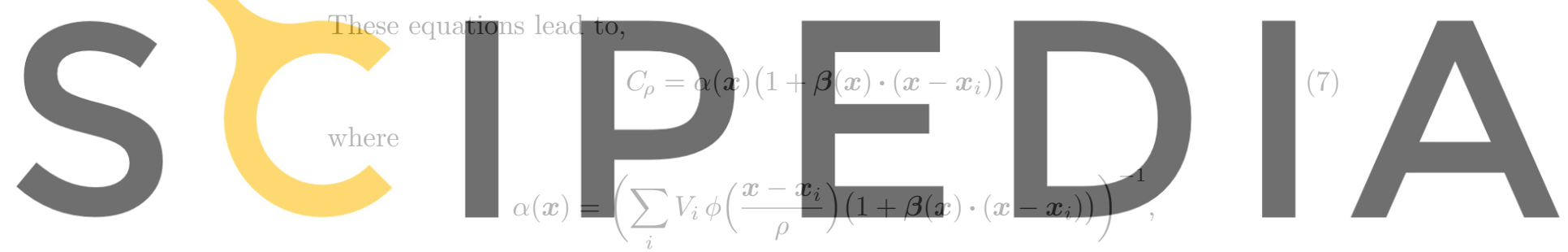

Register for free at 狔ps//www.scipedia.com to download the version without the watermark

$$
\beta(x)=\left(\sum_{i} V_{i} \phi\left(\frac{x-x_{i}}{\rho}\right)\left(x-x_{i}\right)\left(x-x_{i}\right)^{T}\right)^{-1} \sum_{i} V_{i}\left(x_{i}-x\right) \phi\left(\frac{x-x_{i}}{\rho}\right) .
$$

The use of this type of correction ensures that linear functions are perfectly interpolated and their gradients are exactly obtained. A possible way of simplifying the calculation is by using constant, rather than linear, correction. This is equivalent to taking $\boldsymbol{\beta}(\boldsymbol{x})=\mathbf{0}$ in equation (7). However, gradient evaluation using the above expressions is expensive, both in computer memory and time consuming.

Second, the gradient functions are directly amended to ensure that the gradient of a general constant or linear function is correctly evaluated. The corrected gradient is defined as

$$
\widetilde{\boldsymbol{\nabla}} u^{\rho}(\boldsymbol{x})=\sum_{i} V_{i}\left(u\left(\boldsymbol{x}_{i}\right)-u(\boldsymbol{x})\right) \widetilde{\boldsymbol{\nabla}} \phi\left(\frac{\boldsymbol{x}-\boldsymbol{x}_{i}}{\rho}\right)
$$

where

$$
\widetilde{\nabla} \phi\left(\frac{\boldsymbol{x}-\boldsymbol{x}_{i}}{\rho}\right)=\mathbf{L}(\boldsymbol{x}) \boldsymbol{\nabla} \phi\left(\frac{\boldsymbol{x}-\boldsymbol{x}_{i}}{\rho}\right) .
$$

It is clear that equation (8) will ensure that the gradient of a constant function vanishes. The correction matrix $\mathbf{L}(\boldsymbol{x})$ is obtained after imposing the linear consistency 
condition, namely

$$
\sum_{i} V_{i} \widetilde{\boldsymbol{\nabla}} \phi\left(\frac{\boldsymbol{x}-\boldsymbol{x}_{i}}{\rho}\right) \boldsymbol{x}_{i}^{T}=\mathbf{I}
$$

This equation enables the explicit evaluation of the correction term as,

$$
\mathbf{L}(\boldsymbol{x})=\left(\sum_{i} V_{i} \boldsymbol{\nabla} \phi\left(\frac{\boldsymbol{x}-\boldsymbol{x}_{i}}{\rho}\right)\left(\boldsymbol{x}_{i}-\boldsymbol{x}\right)^{T}\right)^{-1}
$$

This corrected gradient, proposed by Bonet and coworkers, is similar to the Renormalized Meshless Derivative (RMD) proposed in [Randles and Libersky (1996); Krongauz and Belytschko (1997); Vila (1999)].

\subsection{Lagrangian $C S P H$}

This section will recall the basics ideas and the notation of the Lagrangian CSPH formulation. The details and the development of this formulation can be found in [Bonet and Kulasegaram (2001)].
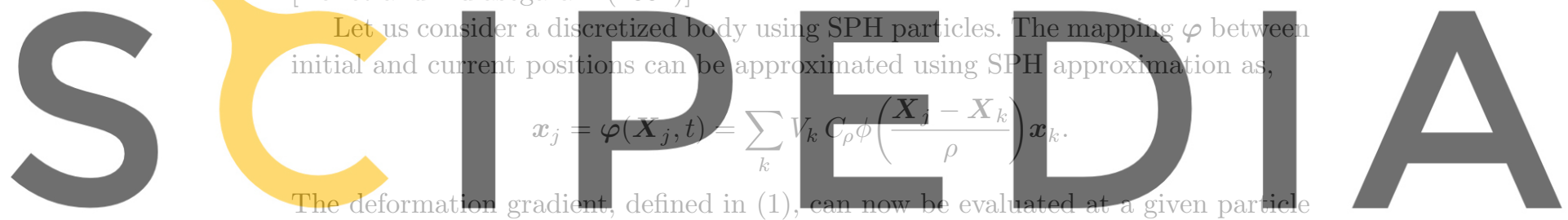

Register for free at https//www.scipedia.com to download the version without the watermark

$$
\mathrm{F}_{j}=\nabla_{0} \varphi=\sum_{k} x_{k} \mathrm{G}_{k}^{T}\left(\boldsymbol{X}_{j}\right),
$$

where $\boldsymbol{\nabla}_{0}$ indicates the gradient respect to the initial configuration, $\boldsymbol{x}_{k}$ is the current position of particle $k$ and where the vectors $\mathbf{G}$ contain the corrected kernel gradients at the initial configuration, that is,

$$
\mathbf{G}_{k}\left(\boldsymbol{X}_{j}\right)=V_{k} \widetilde{\boldsymbol{\nabla}}_{0} \phi\left(\frac{\boldsymbol{X}_{j}-\boldsymbol{X}_{k}}{\rho}\right)
$$

where $\widetilde{\nabla}_{0}$ is a "corrected" gradient to ensure linear completeness as shown in (8).

Once the expression of the deformation gradient is determined the internal forces can be computed using the first Piola-Kirchhoff tensor, $\mathbf{P}$. Thus, the variation of internal forces in a Lagrangian $\mathrm{CSPH}$ formulation in the reference configuration are:

$$
\delta \dot{w}_{\text {int }}=\int_{V^{0}} \mathbf{P}: \delta \dot{\mathbf{F}} d V^{0} \simeq \sum_{j} V_{j}^{0} \mathbf{P}_{j}: \delta \dot{\mathbf{F}}_{j} .
$$

The variation of the virtual deformation gradient emerges from equation (9) as

$$
\delta \dot{\mathbf{F}}_{j}=\sum_{k} \delta \mathbf{v}_{k} \mathbf{G}_{k}^{T}\left(\boldsymbol{X}_{j}\right),
$$


which after substitution into (10) leads to the expression of the internal virtual work

$$
\delta \dot{w}_{\text {int }} \simeq \sum_{j} V_{j}^{0} \mathbf{P}_{j}:\left(\sum_{k} \delta \mathbf{v}_{k} \mathbf{G}_{k}^{T}\left(\boldsymbol{X}_{j}\right)\right)=\sum_{k} \delta \mathbf{v}_{k} \cdot\left(\sum_{j} V_{j}^{0} \mathbf{P}_{j} \mathbf{G}_{k}\left(\boldsymbol{X}_{j}\right)\right) .
$$

With this expression the vector of internal forces corresponding to a certain particle $i$ can be identified as

$$
\mathbf{T}_{i}=\sum_{j} V_{j}^{0} \mathbf{P}_{j} \mathbf{G}_{i}\left(\boldsymbol{X}_{j}\right)
$$

It is important to observe that in (11) the kernel derivatives, $\mathbf{G}_{i}\left(\boldsymbol{X}_{j}\right)$, are fixed in the reference configuration and therefore they do not depend on the current positions of the particles. This bears that corrections are only calculated at the beginning reducing the computational cost.

\subsection{Numerical Example: cylinder bending test with a hyperelastic} material
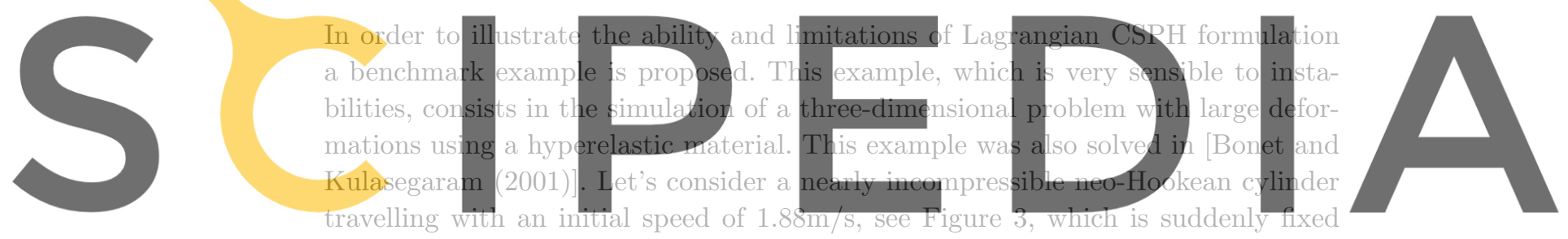

at its base. Homogeneous Dirichlet boundary conditions are directly imposed to

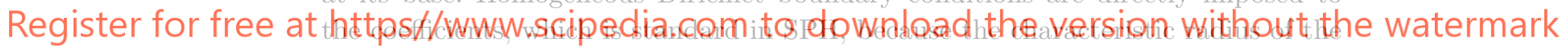

support of the kernel function is small. For larger values of $\rho$ other alternatives may be implemented [Fernandez-Mendez and Huerta (2004)]. The initial radius is $0.32 \mathrm{~m}$ and the length $3.24 \mathrm{~m}$. The shear modulus is taken as $0.3571 \mathrm{MN} / \mathrm{m}^{2}$ and the bulk modulus is $1.67 \mathrm{MN} / \mathrm{m}^{2}$.

The results obtained using a Lagrangian CSPH formulation can be seen in Figure 10. The bar oscillates from initial position to maximum deformation and then back to initial position as expected. The stress component $\sigma_{z z}$ is shown where $z$ is the height component. The cylinder deformation is simulated with good results even in the presence of high tension.

\section{STANDARD UPDATED LAGRANGIAN}

The updated Lagrangian formulation consists of a multiplicative incremental approach as illustrated in Figure 5. The intermediate configuration $\boldsymbol{x}^{r}$ will be the new reference configuration for the next time steps. This means that a new neighbor search must be done in this configuration, $\boldsymbol{x}^{r}$, and that corrections of the kernel and its derivatives must be recalculated.

It is important to observe that the deformation gradient $\mathbf{F}^{r}$ is stored as an internal state variable and only the incremental deformation gradient, $\mathbf{f}$, (i.e. the 


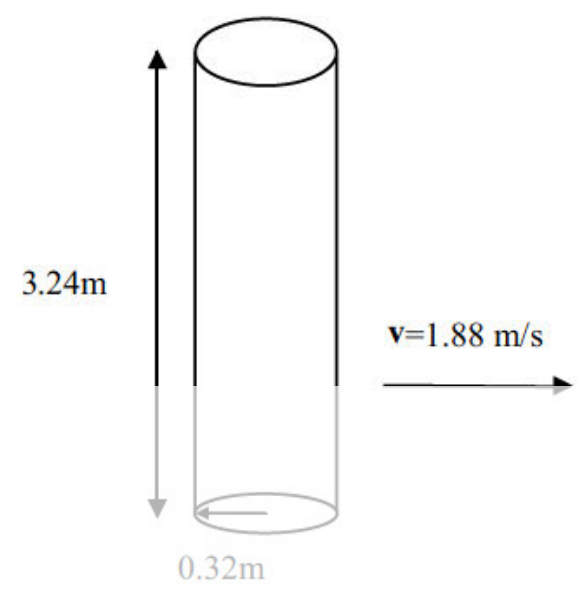

Fig. 3. Cylinder travelling with an initial speed of $1.88 \mathrm{~m} / \mathrm{s}$.
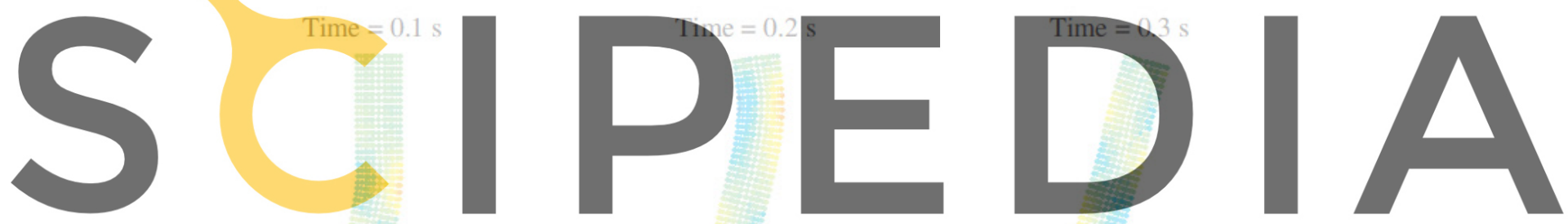

Register for free at https//www.scipedia.com to download the version without the watermark Time $=0.4 \mathrm{~s}$ Time $=0.5 \mathrm{~s}$ Time $=0.6 \mathrm{~s}$
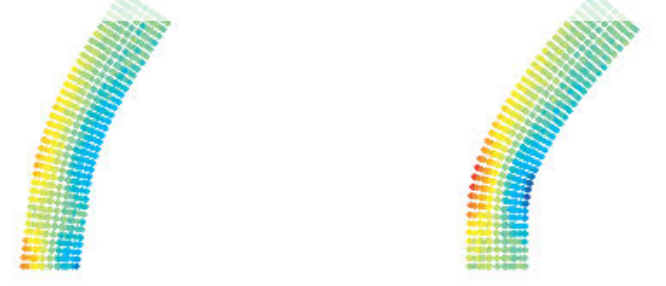

Time $=0.7 \mathrm{~s}$

$$
\text { Time }=0.8 \mathrm{~s}
$$
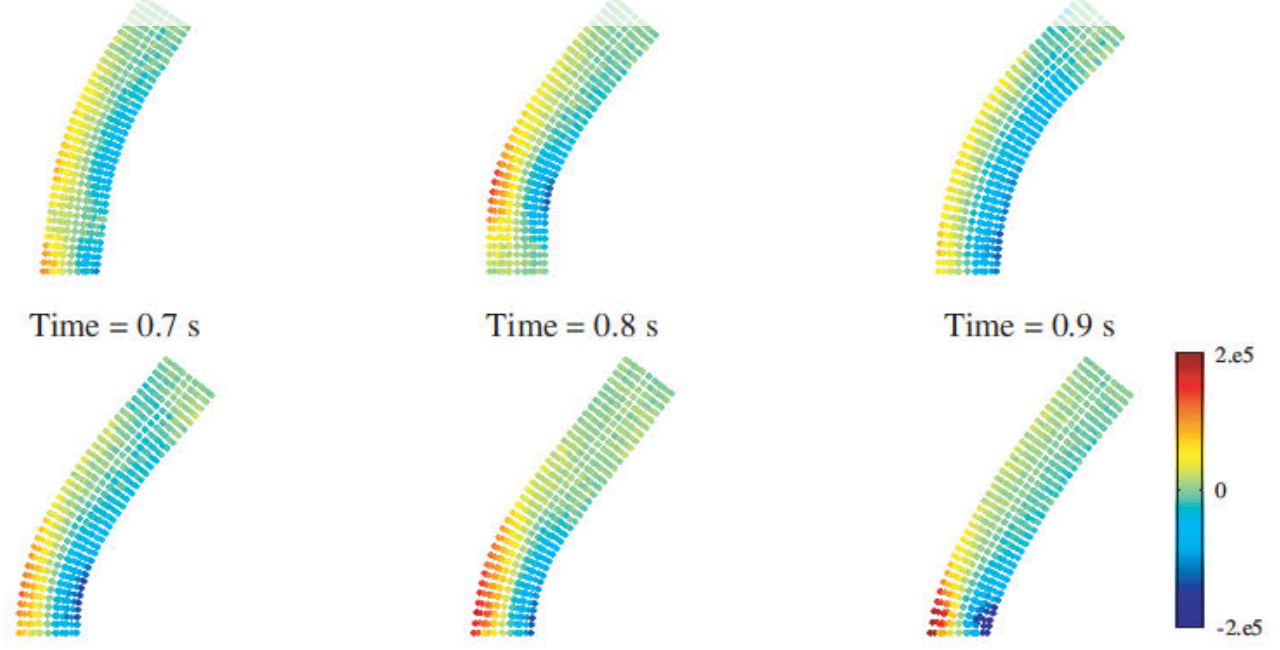

Fig. 4. Total Lagrangian formulation: cylinder bending test. Stress component $\sigma_{z z}(z$ is the height component) is shown. 


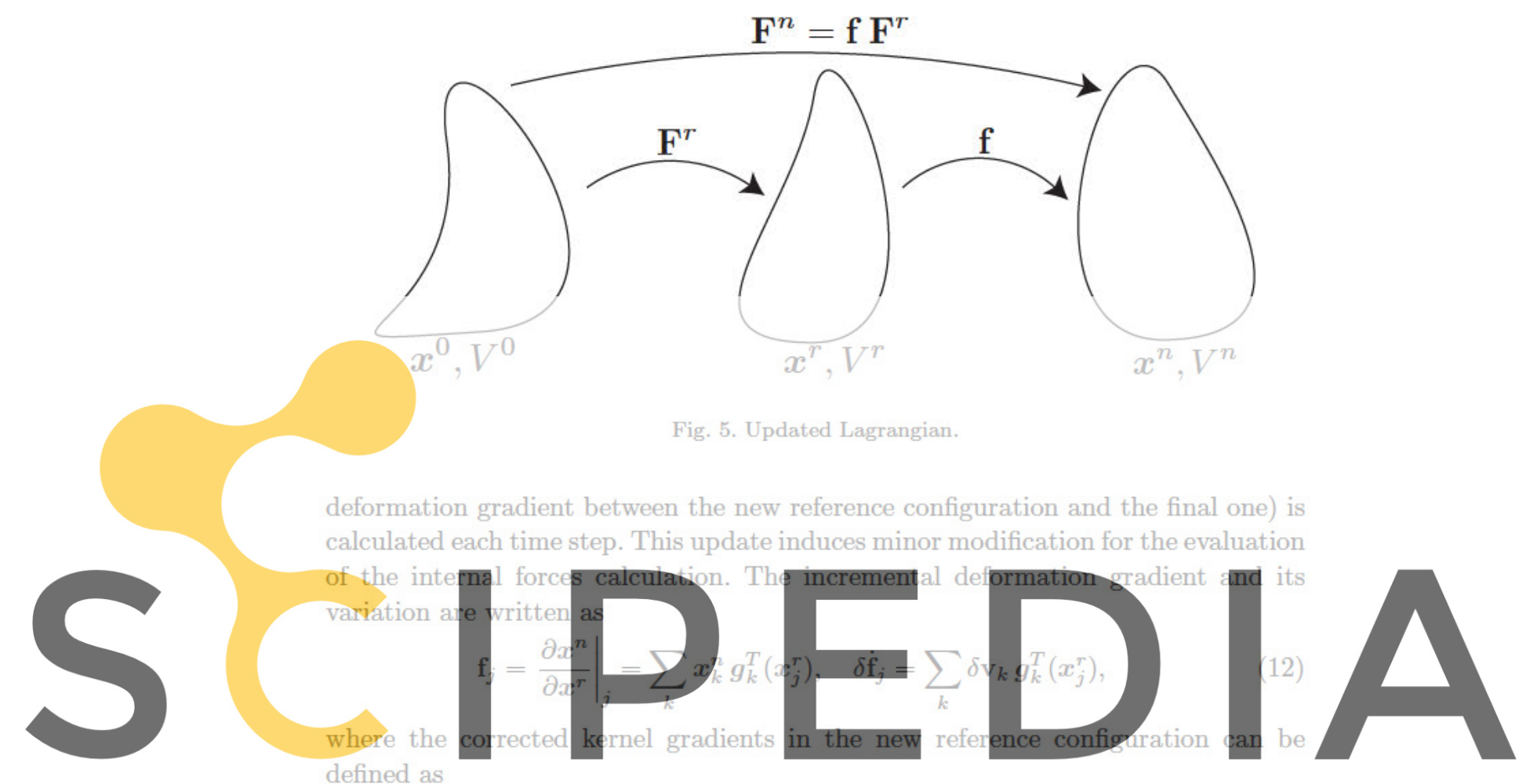

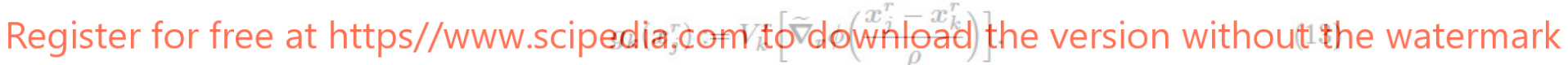

Thus, the variation of internal virtual work expressed in the initial configuration is

$$
\delta \dot{w}_{\text {int }}=\int_{V^{0}} \mathbf{P}^{n}: \delta \dot{\mathbf{F}}^{n} d V^{0} \simeq \sum_{j} V_{j}^{0} \mathbf{P}_{j}^{n}: \delta \dot{\mathbf{F}}_{j}^{n}=\sum_{j} V_{j}^{0} \mathbf{P}_{j}^{n}:\left(\delta \dot{\mathbf{f}}_{j} \mathbf{F}_{j}^{r}\right) .
$$

Recalling the identity $\mathbf{A}: \mathbf{B}=\operatorname{tr}\left(\mathbf{A B}^{T}\right)$, which implies that

$$
\mathbf{P}_{j}^{n}:\left(\delta \dot{\mathbf{f}}_{j} \mathbf{F}_{j}^{r}\right)=\operatorname{tr}\left(\mathbf{P}_{j}^{n}\left[\mathbf{F}_{j}^{r}\right]^{T} \delta \dot{\mathbf{f}}_{j}^{T}\right)=\left(\mathbf{P}_{j}^{n}\left[\mathbf{F}_{j}^{r}\right]^{T}\right): \delta \dot{\mathbf{f}}_{j},
$$

the internal virtual work can be written as

$$
\delta \dot{w}_{\text {int }}=\sum_{j} V_{j}^{0}\left(\mathbf{P}_{j}^{n}\left[\mathbf{F}_{j}^{r}\right]^{T}\right): \delta \dot{\mathbf{f}}_{j}=\sum_{j} V_{j}^{0}\left(\mathbf{P}_{j}^{n}\left[\mathbf{F}_{j}^{r}\right]^{T}\right):\left(\sum_{k} \delta \mathbf{v}_{k} \boldsymbol{g}_{k}^{T}\left(\boldsymbol{x}_{j}^{r}\right)\right)
$$

which can be further simplified using $\mathbf{A}:\left(\mathbf{u} \mathbf{v}^{T}\right)=\mathbf{u} \cdot \mathbf{A v}$ as

$$
\delta \dot{w}_{\text {int }}=\sum_{k} \delta \mathbf{v}_{k} \cdot\left(\sum_{j} V_{j}^{0}\left(\mathbf{P}_{j}^{n}\left[\mathbf{F}_{j}^{r}\right]^{T}\right) \boldsymbol{g}_{k}\left(\boldsymbol{x}_{j}^{r}\right)\right)
$$

and therefore at the current configuration, $x^{n}$, the internal forces vector at a given particle $i$ can be easily evaluated using a reference configuration $x^{r}$ as

$$
\mathbf{T}_{i}^{n, r}=\sum_{j} V_{j}^{0}\left(\mathbf{P}_{j}^{n}\left[\mathbf{F}_{j}^{r}\right]^{T}\right) \boldsymbol{g}_{i}\left(\boldsymbol{x}_{j}^{r}\right) .
$$




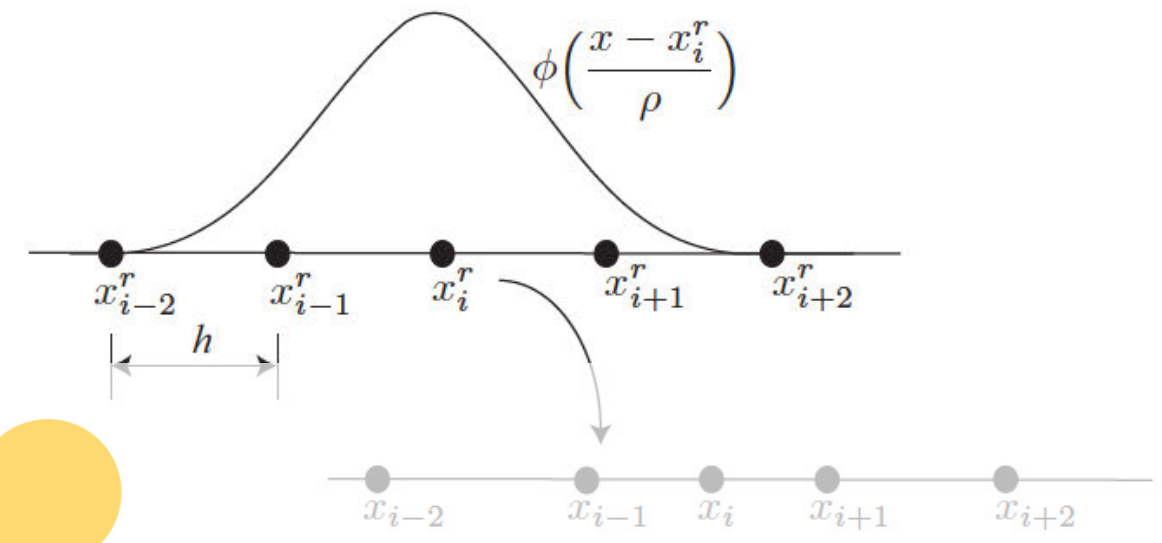

Fig. 6. 1D Updated Lagrangian CSPH.
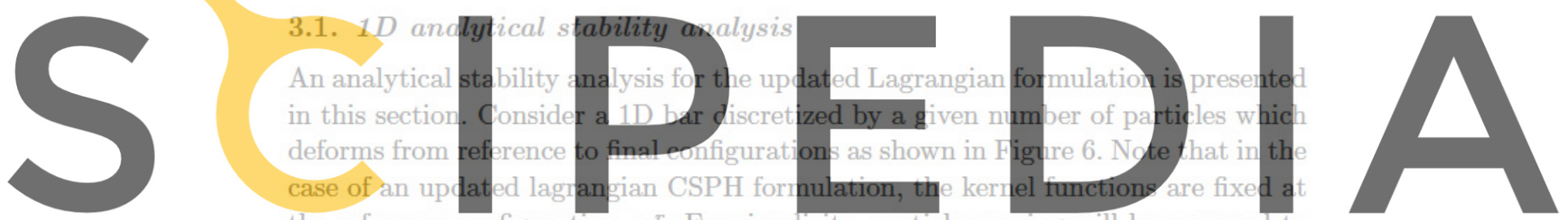

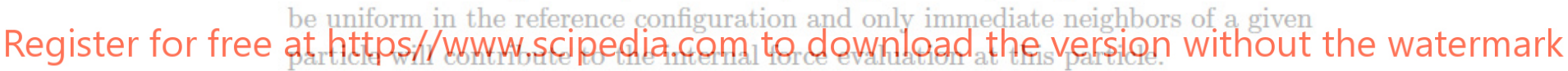

Given the one-dimensional nature of the problem and taking the area as constant and equal to one (in the reference configuration), the deformation gradient is simply given as

$$
\mathrm{F}_{i}=\mathrm{f}_{i} \mathrm{~F}_{i}^{r}=\left(\sum_{j} x_{j} g_{j}\left(x_{i}^{r}\right)\right) \mathrm{F}_{i}^{r} .
$$

For the simple uniformly spaced reference configuration considered, the linearly corrected gradient terms $g$ are simply

$$
g_{i-1}\left(x_{i}^{r}\right)=-\frac{1}{2 h}, g_{i}\left(x_{i}^{r}\right)=0, \text { and } g_{i+1}\left(x_{i}^{r}\right)=\frac{1}{2 h} ;
$$

which upon substitution into (15) leads to

$$
\mathrm{F}_{i}=\frac{x_{i+1}^{r}-x_{i-1}^{r}}{2 h} \mathrm{~F}_{i}^{r} .
$$

Consider now the internal force equation in the current 1D context

$$
\mathrm{T}_{i}^{n, r}=\sum_{j} V_{j}^{0} \mathrm{P}_{j} \mathrm{~F}_{j}^{r} g_{i}\left(x_{j}^{r}\right)=\sum_{j} V_{j}^{n} \sigma_{j} g_{i}\left(x_{j}^{r}\right) / \mathrm{f}_{j} .
$$


Using this identity together with (16) for the gradient functions enables the internal force at point $i$ to be obtained as

$$
\mathrm{T}_{i}=\left(\frac{V_{i-1}^{n} \sigma_{i-1}}{\mathrm{f}_{i-1}}-\frac{V_{i+1}^{n} \sigma_{i+1}}{\mathrm{f}_{i+1}}\right) / 2 h .
$$

The internal force vector is only a function of the current nodal positions via the stress values. Using the linear constitutive relationship, $\sigma_{i}=\kappa\left(J_{i}-1\right)$, the tangent stiffness matrix terms are now easily evaluated to give

$$
\begin{gathered}
K_{i, i}=\frac{V^{n} \kappa}{\left(x_{i}-x_{i-2}\right)^{2}}+\frac{V^{n} \kappa}{\left(x_{i+2}-x_{i}\right)^{2}}, \\
K_{i, i+1}=K_{i, i-1}=0 \\
K_{i, i+2}=\frac{-V^{n} \kappa}{\left(x_{i+2}-x_{i}\right)^{2}}, \quad \text { and } \quad K_{i, i-2}=\frac{-V^{n} \kappa}{\left(x_{i}-x_{i-2}\right)^{2}} .
\end{gathered}
$$

Finally a simple calculation shows that the eigenvalue associated to the alternating eigenvector $(-1)^{i}$ now vanishes as

$$
\sum_{j} K_{i j}(-1)^{j}=0
$$

The above equation implies that this alternating mode is a mechanism instead of a mode with a possible negative eigenvalue as happens in the Eulerian formulation, see [Bonet and Kulasegaram (2001)]. Consequently, the algorithm should be stable but, in the absence of artificial viscosity, undamped oscillations may emerge during the computations.

\subsection{Numerical examples}

\subsubsection{One-dimensional example}

The previous section has proven the existence of mechanisms in the updated Lagrangian formulation. This was also proven for the total Lagrangian formulation in [Bonet and Kulasegaram (2001)]). Next, a 1D numerical test is performed in order to verify whether in these formulations the mechanisms are activated or not.

The total Lagrangian and the updated Lagrangian formulations will be used to solve the elastic 1D bar problem described in Figure 7, see [Dyka and Ingel (1995)]. The bar is fixed at the left end A and the right quarter of the bar is given an initial velocity of $\mathbf{v}_{0}=5 \mathrm{~m} / \mathrm{s}$ thus putting the bar in tension initially. Standard SPH methods cannot solve this problem due to tension instability that immediately develops.

The problem is solved using a uniform distribution of particles. As shown in Figure 7 the CSPH particle distribution is coarse with only 40 uniform particles used in the model. Figure 8 presents the displacement time history of the right end B for the totally Lagrangian formulation and the updated Lagrangian one with 


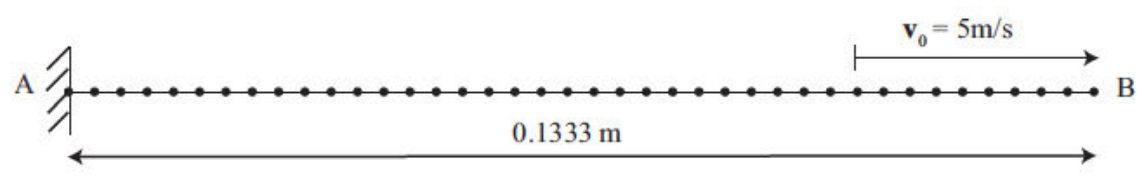

Fig. 7. 1D bar problem statement.
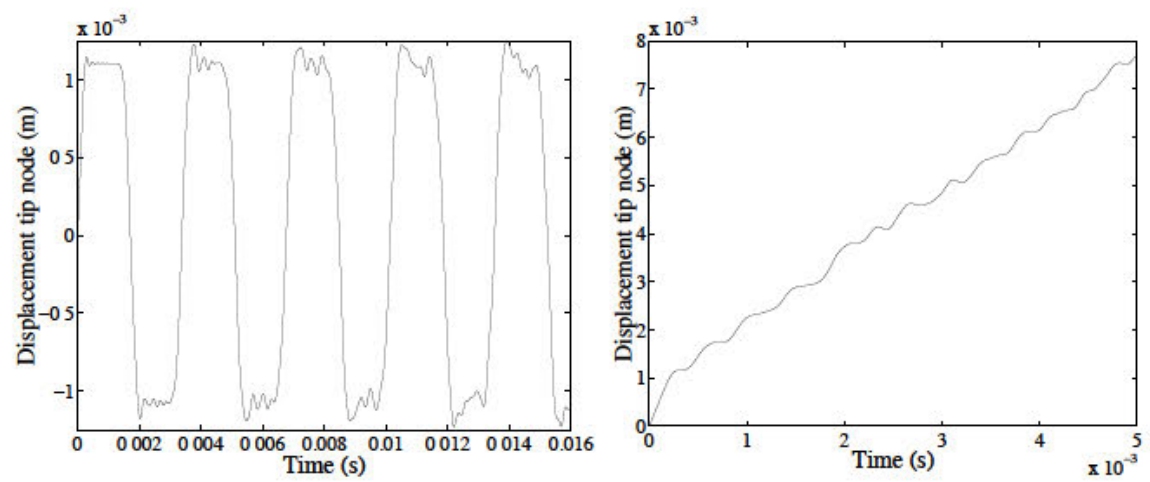

Fig. 8. Displacement history for the right end of the bar (point B). Total Lagrangian (left) and updated Lagrangian (right) formulations.
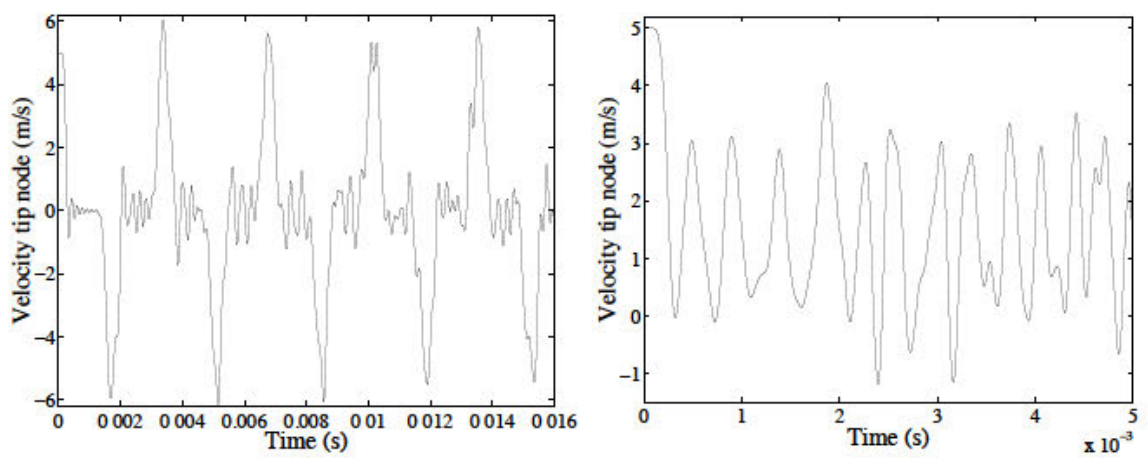

Fig. 9. Velocity history for the right end of the bar (point B). Total Lagrangian (left) and updated Lagrangian (right) formulations.

updates every three time-steps. Figure 9 indicates the predicted time history for the velocity of the right end B. It becomes clear from Figures 8 and 9 that in the updated Lagrangian formulation the mechanisms are activated and they spoil the solution. It seems from this example that performing updates can activate the mechanisms. 

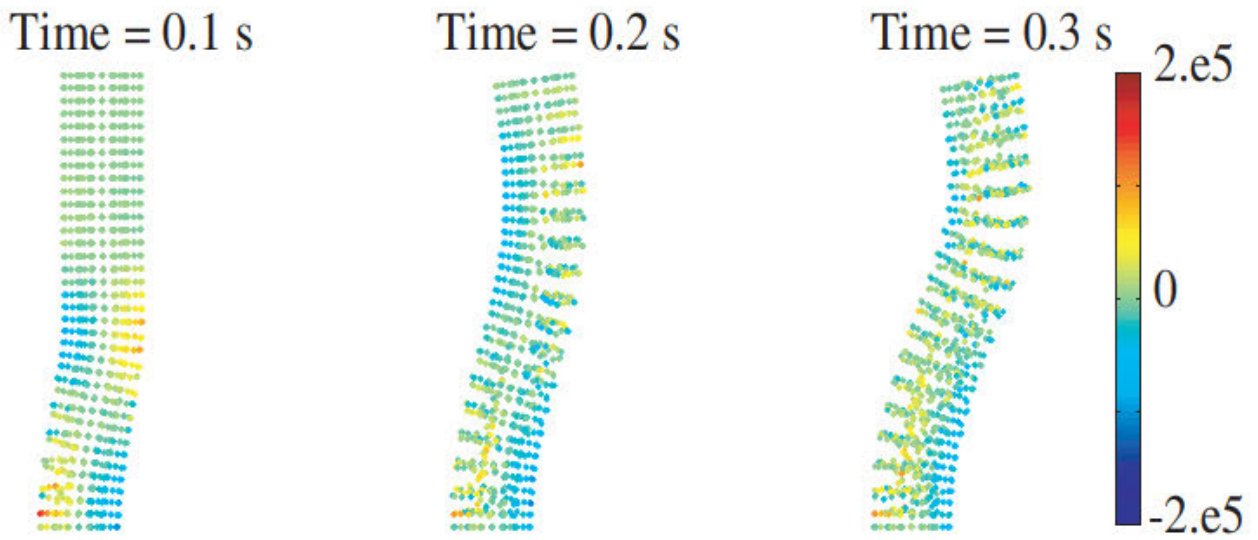

Fig. 10. Updated Lagrangian formulation: cylinder bending test.

\subsubsection{Cylinder bending test with a hyperelastic material}

In fact, if the problem presented in section 2.4 is repeated using a standard updated Lagrangian formulation with updates performed every three time-steps, the results show clearly the mechanisms. Note that these results are very similar to the wellknown tension instabilities, which in this case can not develop, that appear in this example if an Eulerian formulation is employed.

\section{STABILIZED UPDATED LAGRANGIAN}

When an updated Lagrangian formulation must be used, it is necessary to preclude that zero energy modes are activated, or at least eliminate the observed mechanisms. Next, a new stabilization technique is proposed. This method is based on the addition of a higher-order derivative term to the corrected gradient of any function, $\psi$, which is approximated, see (5), with the standard expression:

$$
\psi(\boldsymbol{x})=\sum_{j} N_{j}(\boldsymbol{x}) \psi_{j}
$$

The added term is the difference of two Hessians and it will converge to zero as the particle distribution is refined:

$$
\tilde{\boldsymbol{\nabla}}^{s} \psi\left(\boldsymbol{x}_{i}^{r}\right):=\sum_{j} \psi_{j} \boldsymbol{g}_{j}\left(\boldsymbol{x}_{i}^{r}\right)+\eta\left[\mathcal{H}_{\psi}\left(\boldsymbol{x}_{i}^{r}\right)-\boldsymbol{\nabla}\left(\boldsymbol{\nabla} \psi\left(\boldsymbol{x}_{i}^{r}\right)\right)\right] \boldsymbol{h}
$$

where $\eta$ is a dimensionless stabilization parameter, $h$ is a vector proportional to the interparticle distance and $\mathcal{H}_{\psi}\left(\boldsymbol{x}_{i}^{r}\right)$ represents the Hessian of $\psi$ which in the context of CSPH can be obtained as,

$$
\mathcal{H}_{\psi}\left(\boldsymbol{x}_{i}^{r}\right):=\sum_{k} V_{k}^{r} \psi_{k} \widetilde{\mathcal{H}}_{\phi_{k}}\left(\boldsymbol{x}_{i}^{r}\right)
$$


where $\widetilde{\mathcal{H}}_{\phi_{k}}$ is the linearly corrected Hessian kernel. To obtain linear reproducibility, $\widetilde{\mathcal{H}}_{\phi_{k}}$ is corrected by means of two terms, namely a matrix $\mathcal{B}(\boldsymbol{x})$ and a third order tensor $\mathcal{A}(\boldsymbol{x})$ as,

$$
\widetilde{\mathcal{H}}_{\phi_{k}}\left(\boldsymbol{x}_{i}^{r}\right)=\mathcal{H}_{\phi_{k}}\left(\boldsymbol{x}_{i}^{r}\right)+\delta_{i k} \mathcal{B}\left(\boldsymbol{x}_{i}^{r}\right)+\mathcal{A}\left(\boldsymbol{x}_{i}^{r}\right) \cdot\left(\boldsymbol{x}_{i}^{r}-\boldsymbol{x}_{k}^{r}\right),
$$

where $\mathcal{H}_{\phi_{k}}\left(\boldsymbol{x}_{i}^{r}\right)$ is the Hessian of the kernel function $\phi$, that is,

$$
\mathcal{H}_{\phi_{k}}(\boldsymbol{x})=\nabla\left(\nabla \phi\left(\frac{\boldsymbol{x}-\boldsymbol{x}_{k}}{\rho}\right)\right)
$$

which has an explicit known expression once the kernel $\phi$ is defined. Correction terms $\mathcal{B}$ and $\mathcal{A}$ are determined enforcing that constant and linear functions must have null Hessian, that is,

$$
\sum_{k} V_{k}^{r} \widetilde{\mathcal{H}}_{\phi_{k}}\left(\boldsymbol{x}_{i}^{r}\right)=\mathbf{0} \text { and } \sum_{k} V_{k}^{r} \widetilde{\mathcal{H}}_{\phi_{k}}\left(\boldsymbol{x}_{i}^{r}\right) \boldsymbol{x}_{k}^{r}=\mathbf{0} .
$$

These reproducibility conditions determine the expressions for $\mathcal{B}(\boldsymbol{x})$ and $\mathcal{A}(\boldsymbol{x})$, namely

$$
\begin{gathered}
\mathcal{A}(\boldsymbol{x})=\left[\sum_{k} V_{k}^{r} \boldsymbol{\mathcal { H }}_{\phi_{k}}(\boldsymbol{x})\left(\boldsymbol{x}_{k}^{r}-\boldsymbol{x}\right)^{T}\right]\left[\sum_{k} V_{k}^{r}\left(\boldsymbol{x}_{k}^{r}-\boldsymbol{x}\right)\left(\boldsymbol{x}_{k}^{r}-\boldsymbol{x}\right)^{T}\right]^{-1} \\
\mathcal{B}(\boldsymbol{x})=\frac{1}{V_{i}^{r}}\left[-\sum_{k} V_{k}^{r} \boldsymbol{\mathcal { H }}_{\phi_{k}}(\boldsymbol{x})+V_{k}^{r} \boldsymbol{\mathcal { A }}(\boldsymbol{x}) \cdot\left(\boldsymbol{x}_{k}^{r}-\boldsymbol{x}\right)\right] .
\end{gathered}
$$

Equation (17) can be rewritten using the definition of the Hessian, equation (18), and the gradient, equation (13), as

$$
\sum_{k} \psi_{k} \widetilde{\boldsymbol{g}}_{k}^{s}(\boldsymbol{x})=\sum_{k} \psi_{k} \boldsymbol{g}_{k}(\boldsymbol{x})+\eta\left[\sum_{k} V_{k}^{r} \psi_{k} \widetilde{\mathcal{H}}_{\phi_{k}}(\boldsymbol{x})-\sum_{k} \psi_{k}\left(\sum_{l} \boldsymbol{g}_{k}\left(\boldsymbol{x}_{l}^{r}\right) \cdot \boldsymbol{g}_{l}^{T}(\boldsymbol{x})\right)\right] \boldsymbol{h} .
$$

Hence $\widetilde{\boldsymbol{g}}_{k}^{s}(\boldsymbol{x})$ can be written as:

$$
\widetilde{\boldsymbol{g}}_{k}^{s}(\boldsymbol{x})=\boldsymbol{g}_{k}(\boldsymbol{x})+\eta\left[V_{k}^{r} \widetilde{\mathcal{H}}_{\phi_{k}}(\boldsymbol{x})-\left(\sum_{l} \boldsymbol{g}_{k}\left(\boldsymbol{x}_{l}^{r}\right) \boldsymbol{g}_{l}^{T}(\boldsymbol{x})\right)\right] \boldsymbol{h},
$$

which is the expression that must be introduced, for instance, in (14) to evaluate the internal forces. Equation (19) represents the complete form for the corrected gradient of the kernel, it includes the correction (for reproducibility in the discrete setting —nodal integration-) and stabilization.

\subsection{Numerical examples}

\subsubsection{One-dimensional test}

When this stabilization is introduced in the updated Lagrangian formulation the results for the one-dimensional bar problem introduced in section 3.2.1 change dramatically. In this case, the parameter $\eta$ is taken equal to 0.3 . Figure 11 shows both displacements and velocities for the right end of the bar, which now agree with the total Lagrangian results. It is important to observe that in order to check the performance of this approach an update is done every three time-steps. 

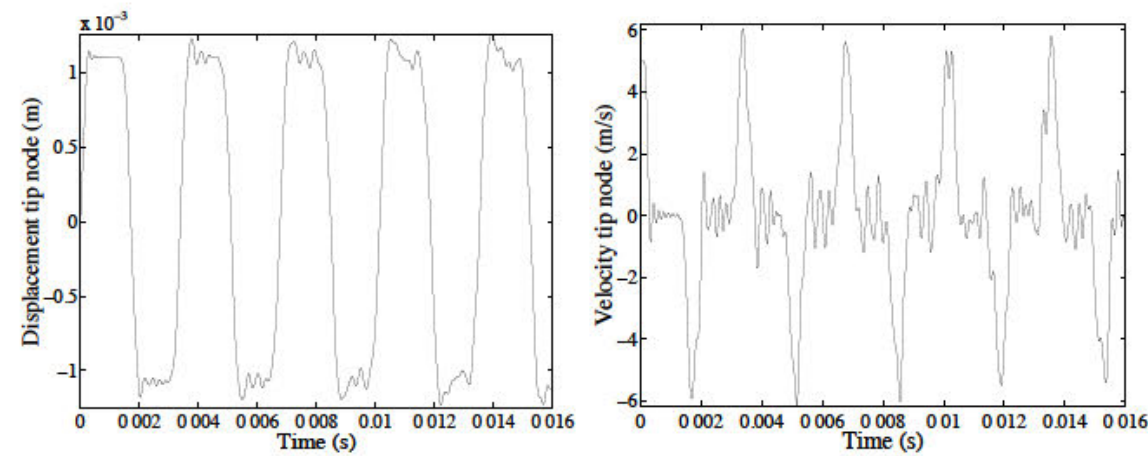

Fig. 11. Displacement (left) and velocity (right) history for the right end of the bar using Hessian's difference stabilization.

\subsubsection{Cylinder bending test with a hyperelastic material}

The oscillating bar presented in section 2.4 is solved using an updated Lagrangian stabilized formulation. These test is particularly demanding for both tension instability (not present in any updated formulation) and spurious mechanisms, that must be suppressed with the stabilization. Moreover, in order to further evaluate the performance of the stabilization updates are activated every three time-steps in order to ensure updates are not activating any mechanisms. Results for $\eta=1.5 \mathrm{can}$ be seen in Figure 12. Time $t=0.9 \mathrm{~s}$ is achieved with 8633 time steps. Thus, 2877 updates have been performed. Good results similar to the ones obtained with the Lagrangian formulation are obtained.

\section{CONCLUSIONS}

In this paper an updated Lagrangian stabilized formulation has been proposed. Its behavior has been tested in a simple one-dimensional synthetic example and in a numerical benchmark test extremely sensible to tensile instability or zero energy modes. The stabilized updated Lagrangian formulation behaves similarly to the total Lagrangian formulation but can encompass problems involving large distortions using updates for the neighbors. Updates are only performed when needed; thus, in practice, the updated formulation has a low computational cost compared to the total Lagrangian one. Moreover, numerical examples show that even for an unreasonable number of updates - inducing, in practice, to a formulation where neighbors a recomputed almost at each time-step - zero (or negative) energy modes are precluded.

\section{References}

T. Belytschko, Y. Guo, W. K. Liu, and S. P. Xiao. A unified stability analysis of meshless particle methods. Internat. J. Numer. Methods Engrg., 48(9):1359-1400, 2000. 


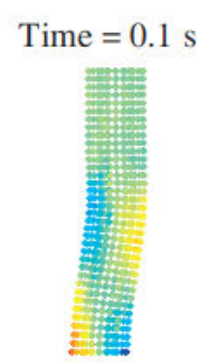

Time $=0.4 \mathrm{~s}$

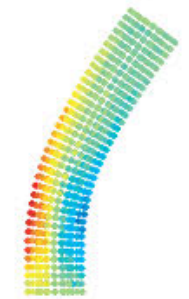

Time $=0.7 \mathrm{~s}$

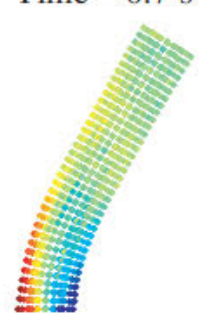

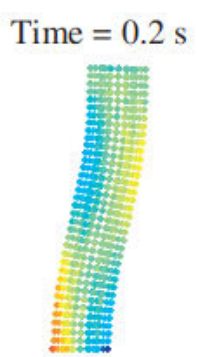

Time $=0.5 \mathrm{~s}$

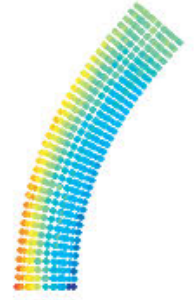

Time $=0.8 \mathrm{~s}$

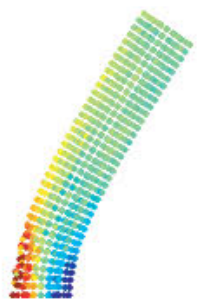

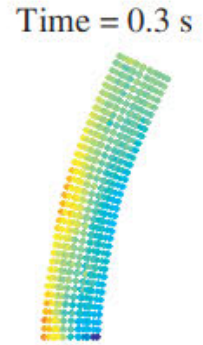

Time $=0.6 \mathrm{~s}$

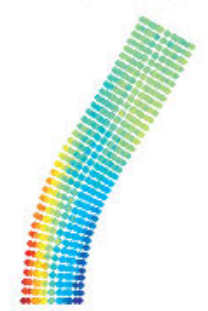

Time $=0.9 \mathrm{~s}$

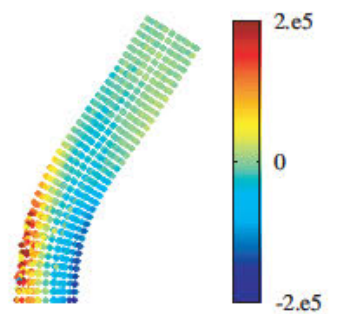

Fig. 12. Stabilized updated Lagrangian formulation: cylinder bending test.

J. Bonet and S. Kulasegaram. Correction and stabilization of smooth particle hydrodynamics methods with applications in metal forming simulations. Int. J. Numer. Methods Eng., 47(6):1189-1214, 2000.

J. Bonet and S. Kulasegaram. Remarks on tension instability of eulerian and lagrangian corrected smooth particle hydrodynamics (CSPH) methods. Int. J. Numer. Methods Eng., 52(11):1203-1220, 2001.

J. Chen, J. Beraun, and T. Carney. A corrective smoothed particle method for boundary value problems in heat conduction. Int. J. Numer. Methods Eng., 46(2):231-252, 1999.

C. T. Dyka and R. P. Ingel. An approach for tension instability in smoothed particle hydrodynamics (SPH). Comput. Struct., 57(4):573-580, 1995.

S. Fernandez-Mendez and A. Huerta. Imposing essential boundary conditions in mesh-free methods. Comput. Methods Appl. Mech. Eng., 193(12-14):1257-1275, 2004.

D. Flanagan and T. Belytschko. A uniform strain hexahedron and quadrilateral with orthogonal hourglass control. Int. J. Numer. Methods Eng., 17(5):679-706, 1981.

J. Gray, J. Monaghan, and R. Swift. SPH elastic dynamics. Comput. Methods Appl. Mech. Eng., 190(49-50):6641-6662, 2001.

A. Huerta, T. Belytschko, S. Fernandez-Mendez, and T. Rabczuk. Meshfree methods. In 
E. Stein, R. de Borst, and T. J. R. Hughes, editors, Encyclopedia of Computational Mechanics, volume 1 Fundamentals, chapter 10, pages 279-309. John Wiley \& Sons, Ltd., Chichester, 2004.

G. R. Johnson and S. R. Beissel. Normalized smoothing functions for SPH impact computations. Int. J. Numer. Methods Eng., 39(16):2725-2741, 1996.

G. R. Johnson, E. H. Petersen, and R. A. Stryk. Incorporation of an SPH option into the EPIC code for a wide-range of high-velocity impact computations. Int. J. Impact Eng., 14(1-4):385-394, 1993.

G. R. Johnson, R. A. Stryk, and S. R. Beissel. SPH for high velocity impact computations. Comput. Methods Appl. Mech. Eng., 139(1-4):347-373, 1996.

Y. Krongauz and T. Belytschko. Consistent pseudo-derivatives in meshless methods. Comput. Methods Appl. Mech. Eng., 146(3-4):371-386, 1997.

L. Libersky, A. Petscheck, T. Carney, J. Hipp, and F. Allahdadi. High strain lagrangian hydrodynamics. J. Comput. Phys., 109(1):67-75, 1993.

M. B. Liu, G. R. Liu, and K. Y. Lam. Constructing smoothing functions in smoothed particle hydrodynamics with applications. J. Comput. Appl. Math., 155(2):263-284, 2003.

W. K. Liu, S. Jun, and Y. F. Zhang. Reproducing kernel particle methods. Int. J. Numer. Methods Fluids, 20(8-9):1081-1106, 1995.

L. Lucy. Numerical approach to testing of the fission hypothesis. Astron. J., 82(12):10131024, 1977.

J. J. Monaghan. Why particle methods work. SIAM J. Sci. Stat. Comput., 3(4):422-433, 1982.

P. W. Randles and L. D. Libersky. Normalized SPH with stress points. Int. J. Numer. Methods Eng., 48(10):1445-1462, 1996.

P. W. Randles and L. D. Libersky. Smoothed particle hydrodynamics: some recent improvements and applications. Comput. Methods Appl. Mech. Eng., 139(1-4):375-408, 1996.

R. Stellingwerf and C. Wingate. Impact modeling with Smooth Particle Hydrodynamics. Int. J. Impact Eng., 14(1-4):707-718, 1993.

J. W. Swegle, D. L. Hicks, and S. W. Attaway. Smoothed particle hydrodynamics stability analysis. J. Comput. Phys., 116(1):123-134, 1995.

J. P. Vila. On particle weighted methods and smooth particle hydrodynamics. Math. Models Methods Appl. Sci., 9(2):161-209, 1999. 